\title{
INFLUÊNCIA DO ETEFON NA DISTRIBUIÇÃO DE NUTRIENTES E CARBOIDRATOS E SOBRE O CRESCIMENTO EM VIDEIRAS JOVENS ${ }^{1}$
}

\author{
PAULA GUERRA SCHENATO 2 , GEORGE WELLINGTON MELO ${ }^{3}$, HENRIQUE PESSOA DOS SANTOS ${ }^{4}$, \\ FLÁVIO BELLO FIALHO ${ }^{5}$, VANEILA FURLANETTO ${ }^{6}$, GUSTAVO BRUNETTO ${ }^{7}$, LIANE TEREZINHA DORNELES $^{8}$
}

RESUMO - O etefon tem sido usado em regiões tropicais e subtropicais do Brasil, onde o crescimento vegetativo das videiras é contínuo, com diferentes finalidades. Entretanto, pouco é conhecido sobre os efeitos deste tratamento na distribuição e acumulação de nutrientes e carboidratos e no crescimento de videiras. Com esta finalidade, plantas jovens do porta-enxerto SO4 foram pulverizadas com solução de etefon (72 mg. $\left.\mathrm{L}^{-1}\right)$. Depois da aplicação, as folhas caídas das plantas tratadas foram coletadas diariamente até que a última folha caiu. Neste momento, as folhas das plantas não tratadas foram desprendidas manualmente e coletadas. No ciclo seguinte, o número de gemas brotadas e o crescimento de folhas (número) e ramos (número e crescimento) foram avaliados em cada planta. Aos 57 dias após a brotação, as videiras foram coletadas e separadas em folhas, ramos, caule e raízes. Cada parte foi secada e submetida à determinação de N, P, K, Ca, Mg e amido. Nas folhas senescentes do primeiro ciclo, foi observada uma redução de N e um aumento de amido e P pelo tratamento com etefon. No ciclo seguinte, as plantas tratadas aumentaram o número de gemas brotadas $(+50 \%)$, mas com redução no crescimento de ramos e folhas. Durante este segundo ciclo, o etefon também aumentou a concentração de $\mathrm{N}$ e $\mathrm{Mg}$ nas folhas e N nas raízes. Entretanto, durante este ciclo, a concentração de amido nas folhas e a quantidade de N, Mg e Ca nos ramos foram reduzidas. Em geral, os resultados enfatizam que o etefon altera a dinâmica de nutrientes e carboidratos, bem como a fisiologia de crescimento no ciclo seguinte da aplicação em videiras.

Termos de indexação: Vitis spp., regulador de crescimento vegetal, etileno, desfolhamento, macronutrientes, amido.

\section{INFLUENCE OF ETHEPHON ON THE DISTRIBUTION OF NUTRIENTS AND CARBOHYDRATES AND ON THE GROWTH IN YOUNG GRAPEVINES}

\begin{abstract}
Ethephon has been used in the tropical and subtropical regions of Brazil, where the vegetative growth of grapevine is continuous, with different finalities. However, little is know about the effects of this treatment on the distribution and accumulation of nutrients and carbohydrates and on the growth of grapevine. For this purpose, young plants of SO4 rootstock were sprayed with ethephon solution $\left(72 \mathrm{mg} . \mathrm{L}^{-1}\right)$. After the application, the dropped leaves of the treated plants were collected daily until the last leaf fell. At this point, the leaves of untreated plants were hand picked. In the next cycle, the number of sprouted buds and growth of leaves (number) and branches (number and length) were evaluated in each plant. Fifty seven days after bud sprout, the plants were collected and separated in leaves, branches, stem and roots. Each part was dried and N, P, K, Ca, Mg and starch were determinate. In the senescence leaves from the first cycle, a reduction of $\mathrm{N}$ and an increase of starch and $\mathrm{P}$ by ethephon treatment were observed. In the following cycle, the treated plants have increased the number of bud sprouts $(+50 \%)$ but with a reduced growth of branches and leaves. During this second cycle the ethephon also increased the concentration of $\mathrm{N}$ and $\mathrm{Mg}$ in leaves and $\mathrm{N}$ in roots. However, during this cycle the concentration of starch in leaves and amount of N, K and $\mathrm{Ca}$ in branches were reduced. In general, the results emphasized that ethephon changes the dynamic of nutrient and carbohydrates as well as the physiology of growth in the following cycle after its application in grapevines.
\end{abstract}

Index terms: Vitis spp., growth regulator, ethylene, defoliation, macronutrients, starch.

\footnotetext{
1 Trabalho (062-06). Recebido em 11-05-2006. Aceito para publicação em: 19-04-2007. Trabalho financiado pela Embrapa Uva e Vinho.

${ }^{2}$ Bióloga, Mestre em Fitotecnia-Fitopatologia, Bento Gonçalves-RS. E-mail: pgschenato@yahoo.com.br.

${ }^{3}$ Engenheiro Agrônomo, Doutor em Ciência do Solo, Pesquisador da Embrapa Uva e Vinho, Caixa Postal 130, Cep: 95700-000, Bento Gonçalves, RS. E-mail: george@cnpuv.embrapa.br (Autor para correspondência).

${ }^{4}$ Engenheiro Agrônomo, Doutor em Fisiologia Vegetal, Pesquisador da Embrapa Uva e Vinho, Bento Gonçalves, RS. E-mail: henrique@cnpuv.embrapa.br

${ }^{5}$ Engenheiro Agrônomo, Doutor em Engenharia Agrícola e Biológica, Pesquisador da Embrapa Uva e Vinho, Bento Gonçalves, RS. E-mail: bello@cnpuv.embrapa.br.

${ }^{6}$ Bióloga, estudante do Curso de Especialização em Agricultura Orgânica da Universidade de Caxias do Sul, Caxias do Sul, RS. E-mail: vaneila@cnpuv.embrapa.br.

${ }^{7}$ Engenheiro Agrônomo, Mestre em Ciência do Solo, Doutorando do Programa de Pós-Graduação em Ciência do Solo da Universidade Federal de Santa Maria, Santa Maria, RS. Bolsista de doutorado do CNPq. E-mail: brunetto@cnpuv.embrapa.br.

${ }^{8}$ Engenheira Agrônoma, Doutora em Botânica, Professora do Departamento de Ciência Exatas e da Natureza, Universidade de Caxias do Sul, CARVI, Bento Gonçalves, RS. E-mail: 1tdornel@ucs.br.
} 


\section{INTRODUÇÃO}

Nas videiras cultivadas em regiões temperadas do Brasil, em geral, a diminuição da temperatura nos meses de inverno estimula a síntese de ácido abscísico nas folhas. Esse hormônio vegetal produz células suberosas que formam um septo entre a base do pecíolo e a lâmina foliar, causando a senescência das folhas e a acumulação de carboidratos e nutrientes nos órgãos de reserva da videira (Pommer, 2003). Por outro lado, nas regiões tropicais, o crescimento vegetativo da videira é contínuo, resultando em plantas nos diversos estádios de desenvolvimento ao longo do ano (Pszczolkowski, 1984). Este crescimento ocorre, principalmente, nas gemas distais, sendo as restantes inibidas pela dominância apical e restringindo o número total de brotações por planta. Isto ocorre porque não há repouso em condições de temperaturas elevadas, sendo este, necessariamente, induzido por déficit hídrico ou por tratamentos com reguladores de crescimento, para facilitar a condução do manejo destas plantas em condições tropicais.

O pequeno número de brotos e a sua desuniformidade de crescimento têm afetado a produtividade dos vinhedos nas regiões de clima tropical e subtropical (Mashima, 2000). Na busca por alternativas que aumentem a produtividade desses vinhedos, tem-se usado a aplicação de ácido 2-cloroetil-fosfônico (etefon), substância química que libera lentamente o regulador de crescimento etileno, na forma gasosa, conseqüentemente, provocando mudanças no metabolismo da planta. O etileno é um dos principais indutores de abscisão de órgãos em espécies vegetais. Isto fica evidente no processo de senescência foliar, onde o ácido abscísico interfere indiretamente pelo aumento da síntese de etileno (Taiz \& Zeiger, 2004).

$O$ etefon tem sido usado como um produto eficaz para aumento de brotação de gemas em condições tropicais. Aplicações sucessivas em videiras provocam o aumento da fertilidade e estimulam a brotação das gemas de ciclos anteriores, aumentando a produtividade da cultura (Albuquerque \& Dantas, 2002; Fracaro et al., 2004a; Fracaro at al., 2004b. Além disso, a aplicação via foliar de etefon em videiras tem-se mostrado eficaz no aumento do crescimento e diâmetro dos ramos (Fracaro \& Pereira, 2004), e no aumento do comprimento, largura e peso dos cachos (Pereira \& Fracaro, 2004). Esses resultados podem estar associados à maior mobilização de nutrientes e carboidratos das folhas para órgãos, como ramos, caule e raízes, os quais são redistribuídos para as partes em crescimento no ciclo vegetativo seguinte, fenômeno comum na videira (Conradie, 1991; Zapata et al., 2004; Brunetto et al., 2005).

De acordo com Fracaro \& Boliani (2001), a melhoria na brotação pelo tratamento com etefon pode ocorrer devido à translocação de nutrientes das folhas para as gemas. Entretanto, até o presente momento, não se dispõe de trabalhos que relacionam, de forma conjunta, a aplicação de etefon em videiras e a sua interferência na dinâmica interna de nutrientes e carboidratos.

O presente trabalho teve como objetivo estimar, de forma conjunta, a influência do etefon sobre a distribuição de nutrientes e carboidratos de reserva sobre o crescimento em videiras jovens.

\section{MATERIAL E MÉTODOS}

O trabalho foi conduzido em casa de vegetação da Embrapa Uva e Vinho, durante o período de abril a outubro de 2003. O experimento constou da avaliação de plantas do portaenxerto de videira SO4 (Vitis berlandierix Vitis riparia), obtidos por meio de propagação vegetativa (estaquia), com seis meses de idade. O cultivo das plantas foi feito em vasos (3 L) contendo solo Argissolo Vermelho-Amarelo (Embrapa, 1999). O solo foi coletado em área não cultivada no município de Caxias do Sul (RS) e apresentava os seguintes atributos: argila 460 g. $\mathrm{kg}^{-1}$; massa orgânica 0,6 g.kg-1; $; \mathrm{pH}-\mathrm{H}_{2} \mathrm{O} 4,9$; índice SMP 5,3; Ca trocável 4,3 mmol $\cdot \mathrm{dm}^{-3} ; \mathrm{Mg}$ trocável 4,0 $\mathrm{mmol}_{\mathrm{c}} \cdot \mathrm{dm}^{-3} ; \mathrm{Al}$ trocável 4,3 $\mathrm{mmol}_{\mathrm{c}} \cdot \mathrm{dm}^{-3} ; \mathrm{P}$ disponível $0,4 \mathrm{mg} \cdot \mathrm{dm}^{-3}$ (Mehlich 1), e K trocável $1,9 \mathrm{mmol}_{\mathrm{c}} \cdot \mathrm{dm}^{-3}$.

Os tratamentos usados foram sem e com a aplicação de etefon. No tratamento sem aplicação de etefon, as videiras receberam a aplicação de $10 \mathrm{~mL}$ de água destilada. Por outro lado, no tratamento com aplicação, as videiras foram submetidas à aplicação de $10 \mathrm{~mL}$ de uma solução contendo $72 \mathrm{mg} . \mathrm{L}^{-1} \mathrm{de}$ ingrediente ativo. Os tratamentos foram aplicados sobre as folhas através de pulverizador manual, uma única vez (02-04$03)$.

O delineamento experimental usado foi inteiramente casualizado, com 10 repetições. Depois da aplicação dos tratamentos, diariamente, foram coletadas as folhas desprendidas das plantas do tratamento com a aplicação de etefon. Aos 21 dias após a aplicação, houve desfolhamento total das plantas (23-04-03). Nesta data, foram coletadas todas as folhas do tratamento sem a aplicação, as quais não apresentaram desprendimento natural durante este período. Estas folhas são referidas neste trabalho como folhas de primeiro ciclo. Depois de 110 dias da aplicação dos tratamentos (21-07-03), as videiras foram podadas, permanecendo três gemas vegetativas por planta. Todo o material resultante da poda foi eliminado. Após a poda, as videiras foram mantidas em casa de vegetação, com temperatura controlada em torno de $20^{\circ} \mathrm{C}$.

A partir do início da brotação do segundo ciclo, 131 dias após a aplicação dos tratamentos (11-08-03), foi avaliado o número de gemas brotadas (gemas que emitiram ramos), o número de ramos e de folhas por planta. Aos 57 dias após o início da brotação (07-10-03), as videiras foram retiradas dos vasos e separadas em folhas, ramos, caule e raízes. Estas últimas foram lavadas com $\mathrm{HCl}$ 0,5 mol. $\mathrm{L}^{-1}$ e água destilada para a retirada dos resíduos de solo. Todas as partes das videiras foram secadas em estufa com ar forçado, a $60^{\circ} \mathrm{C}$, até atingir peso constante. A seguir, determinaram-se a massa seca, a concentração de N, P, K, Ca e $\mathrm{Mg}$, segundo metodologia proposta por Tedesco et al. (1995), e a de amido, conforme método enzimático descrito por Santos et al. (2004).

Os resultados obtidos foram submetidos à análise de variância e, quando da significância, foram submetidos ao teste t, tomando como base os níveis de significância maiores que 95\% ( $<<0,05)$, utilizando o software R (http://www.r-project.org/). 


\section{RESULTADOS E DISCUSSÃO}

As videiras submetidas à aplicação de etefon apresentaram folhas de primeiro ciclo com concentração e quantidade de nitrogênio menores que as videiras do tratamento sem aplicação do produto (Tabela 1). Esses dados sugerem que o etefon pode ter favorecido a maior redistribuição de nitrogênio destas folhas para as raízes. Essa hipótese pode ser suportada pela maior concentração desse elemento encontrada nas raízes do segundo ciclo (Tabela 3). Além disso, a aplicação de etefon aumentou a concentração e a quantidade de fósforo nas folhas de primeiro ciclo (Tabela 1). Considerando que o etefon possui fósforo em sua formulação (ácido 2-cloroetil-fosfônico), este resultado sugere que este elemento permaneceu nas folhas após a aplicação.

O tratamento com etefon, no primeiro ciclo vegetativo, também promoveu aumento na concentração e quantidade de amido nas folhas, comparativamente ao tratamento sem aplicação (Tabela 1). Isso, possivelmente, se deve à antecipação da senescência e abscisão das folhas provocada pelo etileno (Taiz \& Zeiger, 2004), restringindo a quantidade de carboidratos mobilizada e distribuída para os órgãos de reserva da videira. De acordo com Marinho et al. (2001), a desfolha antecipada e drástica induzida pelo etefon, aliada à mobilidade de cada nutriente podem ser considerados importantes fatores na definição das alterações nutricionais em plantas tratadas com este produto.

TABELA 1 - Efeito da aplicação de etefon sobre a produção de massa seca, concentração e teor total de nutrientes e amido nas folhas de primeiro ciclo das videiras do porta-enxerto SO4. Bento Gonçalves- RS, 2006.

\begin{tabular}{|c|c|c|c|c|}
\hline Variável $^{1}$ & Sem etefon & Com etefon & Sem etefon & Com etefon \\
\hline \multicolumn{5}{|c|}{ g.planta $^{-1}$} \\
\hline Massa seca & 1,47 & 1,39 & - & - \\
\hline & \multicolumn{2}{|c|}{ Concentração } & \multicolumn{2}{|c|}{ Teor total $^{2}$} \\
\hline & \multicolumn{2}{|c|}{$\mathrm{g} \cdot \mathrm{kg}^{-1}$} & \multicolumn{2}{|c|}{ mg.folha ${ }^{-1}$} \\
\hline Nitrogênio & $1,30 \mathrm{a}$ & $1,12 b$ & 19,21 a & $15,45 \mathrm{~b}$ \\
\hline Fósforo & $0,13 b$ & $0,40 \mathrm{a}$ & $1,92 \mathrm{~b}$ & $5,58 \mathrm{a}$ \\
\hline Potássio & 1,16 & 1,27 & 17,23 & 17,40 \\
\hline Cálcio & 1,86 & 1,74 & 27,23 & 24,27 \\
\hline Magnésio & 0,40 & 0,40 & 5,89 & 5,60 \\
\hline Amido & $0,06 \mathrm{~b}$ & $0,25 \mathrm{a}$ & $1,00 \mathrm{~b}$ & $3,62 \mathrm{a}$ \\
\hline
\end{tabular}

'Para a mesma variável, médias seguidas de letras diferentes na linha diferem entre si, pelo teste $\mathrm{t}(p \leq 0,05)$.

${ }^{2}$ Teor total de nutrientes considerando-se todas as folhas de cada videira.

No segundo ciclo vegetativo, a aplicação de etefon aumentou o número de gemas brotadas, refletindo num maior número de ramos por planta (Tabela 2). Isso pode ter ocorrido porque o etefon influencia na paradormência, reduzindo a dominância apical e estimulando a brotação das gemas laterais (Bautista et al., 1991; Fracaro \& Boliani, 2001; Albuquerque \& Dantas, 2002; Fracaro \& Pereira, 2004; Fracaro et al., 2004b). Entretanto, apesar do maior número de ramos, o número de folhas de segundo ciclo foi semelhante nas videiras dos dois tratamentos. Isto se deve ao fato de que, nas videiras submetidas à aplicação de etefon, o crescimento individual dos ramos foi menor (Tabela 2), o que restringiu a emissão de novas folhas. Esta restrição de crescimento, provocada pela aplicação de etefon, também fica evidente nos dados de massa seca de folhas e ramos (Tabela 2), e pode ser uma conseqüência do desequilíbrio da relação fonte/dreno das plantas tratadas. Essa hipótese é corroborada pelo maior estímulo à brotação lateral e conseqüente incremento na competição pelas reservas das plantas. Além disso, as plantas tratadas tiveram restrição no acúmulo de reservas, principalmente de carbono, pelo maior estímulo da senescência foliar e restrição na mobilização de carboidratos no final do ciclo anterior, como explicado anteriormente. Nossos resultados de crescimento diferem dos obtidos por Fracaro \& Pereira, 2004, e Fracaro, 2004, possivelmente pelo fato de as plantas-controle, em condições temperadas, não continuarem vegetando no período de inverno e, portanto, não consumindo as reservas para a brotação do próximo ciclo. Com isso, o que mais se difere é o número de ramos competindo pelo mesmo conteúdo de reservas e não por diferenças em quantidades de reservas.

TABELA 2 - Efeito da aplicação de etefon sobre o número de gemas brotadas e folhas, número e comprimento dos ramos, e produção de massa seca no segundo ciclo das videiras do porta-enxerto SO4. Bento Gonçalves- RS, 2006.

\begin{tabular}{llcc}
\hline \multicolumn{1}{c}{ Variável $^{1}$} & & Sem etefon & Com etefon \\
\hline Gemas Brotadas & & $2,0 \mathrm{~b}$ & $3,0 \mathrm{a}$ \\
Folhas & & 21,2 & 24,3 \\
Ramos (número) & $2,0 \mathrm{~b}$ & $3,0 \mathrm{a}$ \\
Ramos (comprimentos, cm) & & $71,7 \mathrm{a}$ & $39,7 \mathrm{~b}$ \\
\hline \multicolumn{3}{c}{} & \multicolumn{2}{c}{ g.planta $^{-1}$} \\
\hline \multirow{2}{*}{ Massa seca } & Parte da videira & $4,64 \mathrm{a}$ & $3,70 \mathrm{~b}$ \\
& Folhas & $2,70 \mathrm{a}$ & $1,88 \mathrm{~b}$ \\
& Ramos & 1,34 & 1,44 \\
& Caule & 6,02 & 5,46 \\
\hline
\end{tabular}

${ }^{1}$ Médias seguidas de letras diferentes na linha diferem entre si, pelo teste $\mathrm{t}(p \leq 0,05)$.

A concentração de nitrogênio e magnésio foi maior apenas nas folhas de segundo ciclo das plantas que receberam etefon (Tabela 3). Considerando que essas plantas receberam etefon no ciclo anterior, esse resultado sugere que o etefon pode ter favorecido a translocação e o acúmulo desses nutrientes para os órgãos de reserva, como sugerido por Fracaro \& Boliani (2001), tornando-as prontamente disponíveis para o crescimento do ciclo seguinte. Entretanto, esse aumento pode também ser relacionado às diferenças de tamanho médio de folhas (compare a diferença entre número e massa foliar dos tratamentos na Tabela 2). Essa menor proporção de massa foliar que as videiras tratadas com etefon apresentaram também, limitou as diferenças significativas na quantidade total de $\mathrm{N}$ e $\mathrm{Mg}$ nas folhas de segundo ciclo, apesar do contraste em concentração (Tabela 2).

Nos ramos do segundo ciclo, a concentração de nitrogênio, potássio e cálcio foi igual nas videiras dos dois tratamentos (Tabela 3). Entretanto, pelo fato de os ramos das plantas que receberam etefon, estarem com menor massa seca (Tabela 2), tiveram menor quantidade total desses elementos (Tabela 3). Essas alterações nutricionais com a aplicação de etefon podem ser explicadas em função da desfolha e da mobilidade 
TABELA 3 - Efeito da aplicação de etefon sobre a concentração e o teor total de nutrientes e amido em diferentes partes das videiras coletadas no segundo ciclo do porta-enxerto SO4. Bento Gonçalves, RS, 2006.

\begin{tabular}{|c|c|c|c|c|c|}
\hline Variável $^{1}$ & $\begin{array}{c}\text { Parte da } \\
\text { videira }\end{array}$ & Sem etefon & Com etefon & Sem etefon & Com etefon \\
\hline & & \multicolumn{2}{|c|}{ Concentração } & \multicolumn{2}{|c|}{ Teor total } \\
\hline & & \multicolumn{2}{|c|}{ g. $\mathrm{kg}^{-1}$} & \multicolumn{2}{|c|}{ mg.planta ${ }^{-1}$} \\
\hline \multirow{4}{*}{ Nitrogênio } & Folhas & $1,54 \mathrm{~b}$ & $1,77 \mathrm{a}$ & 70,29 & 65,42 \\
\hline & Ramos & 0,55 & 0,54 & 14,89 a & $10,27 b$ \\
\hline & Caule & 0,46 & 0,49 & 6,22 & 7,01 \\
\hline & Raízes & $0,80 \mathrm{~b}$ & $0,88 \mathrm{a}$ & 47,68 & 47,92 \\
\hline \multirow{4}{*}{ Fósforo } & Folhas & 0,10 & 0,11 & 4,45 & 3,98 \\
\hline & Ramos & 0,03 & 0,04 & 0,89 & 0,87 \\
\hline & Caule & 0,02 & 0,02 & 0,27 & 0,31 \\
\hline & Raízes & 0,06 & 0,06 & 3,46 & 3,54 \\
\hline \multirow{4}{*}{ Potássio } & Folhas & 1,32 & 1,32 & 61,39 & 48,80 \\
\hline & Ramos & 1,16 & 1,12 & 31,71 a & $21,73 \mathrm{~b}$ \\
\hline & Caule & 0,56 & 0,49 & 7,57 & 7,45 \\
\hline & Raízes & 0,64 & 0,68 & 38,73 & 36,95 \\
\hline \multirow{4}{*}{ Cálcio } & Folhas & 0,99 & 1,04 & 45,90 & 38,73 \\
\hline & Ramos & 0,41 & 0,41 & 10,96 a & $7,77 \mathrm{~b}$ \\
\hline & Caule & 0,41 & 0,39 & 5,54 & 5,67 \\
\hline & Raízes & 0,50 & 0,48 & 30,36 & 26,22 \\
\hline \multirow{4}{*}{ Magnésio } & Folhas & $0,31 \mathrm{~b}$ & $0,36 a$ & 14,39 & 13,48 \\
\hline & Ramos & 0,10 & 0,12 & 2,73 & 2,17 \\
\hline & Caule & 0,08 & 0,08 & 1,10 & 1,20 \\
\hline & Raízes & 0,09 & 0,09 & 5,48 & 4,85 \\
\hline \multirow{4}{*}{ Amido } & Folhas & $0,82 \mathrm{a}$ & $0,21 \mathrm{~b}$ & $45,87 \mathrm{a}$ & $7,71 \mathrm{~b}$ \\
\hline & Ramos & 1,26 & 1,21 & 39,24 & 26,86 \\
\hline & Caule & 2,09 & 2,24 & 33,03 & 38,11 \\
\hline & Raízes & 5,05 & 5,42 & 343,52 & 368,83 \\
\hline
\end{tabular}

'Para a mesma variável, médias seguidas de letras diferentes na linha diferem entre si, pelo teste $\mathrm{t}(p \leq 0,05)$.

dos nutrientes, onde a desfolha drástica promove redução no acúmulo das reservas de nutrientes em plantas tratadas com etefon no ciclo anterior (Marinho et al., 2001).

Tanto a concentração como a quantidade de amido nas folhas de segundo ciclo das plantas tratadas foram menores, comparativamente às plantas sem etefon (Tabela 3). Esses resultados salientam que a demanda por carbono é maior nas videiras tratadas, por possuírem maior número de ramos (Tabela 2), restringindo o acúmulo transitório nas folhas. Esse é um comportamento típico do metabolismo do carbono em folhas, onde o amido atua como um regulador entre a demanda (mobilização para tecidos em crescimento e de reserva) e a fonte (fotossíntese) (Majerowicz, 2004). Esses resultados sugerem que a aplicação de etefon pode proporcionar maior exigência de manejo de raleio de brotos, para que não se tenha um comprometimento do crescimento e, conseqüentemente, do potencial de produção das plantas tratadas.

\section{CONCLUSÕES}

1- O etefon, logo após a aplicação, apresentou influência no metabolismo de reservas foliares, acelerando a mobilização de nitrogênio e restringindo o fluxo de carboidratos, pelo processo de senescência.

2- No ciclo seguinte ao tratamento, o etefon estimulou a brotação de gemas. Entretanto, restringiu o vigor de crescimento de folhas e ramos sem grandes influências na dinâmica de nutrientes, exceto pelo aumento na concentração foliar de nitrogênio e magnésio e nitrogênio radicular.

\section{REFERÊNCIAS}

ALBUQUERQUE, T. C. S.; DANTAS, B. F. Uso do ethephon em videiras da cv. Itália: I. Efeito sobre a brotação e fertilidade de gemas. In: SIMPÓSIO MINEIRO DE VITICULTURA E ENOLOGIA, 1., 2002, Andradas, MG. Viticultura e enologia: atualizando conceitos. Caldas: EPAMIG - FECD, 2002. p. 305309.

BAUTISTA, A. D.; VARGAS, G.; COLMENARES, J. C. Influencia del etefon sobre la brotacion y fertilidad de tres cultivares de vid. Agronomia Tropical, Maracay, v. 41, p. 225-235, 1991.

BRUNETTO, G.; KAMINSKI, J.; MELO, G. W. B.; GATIBONI, L. C.; URQUIAGA, S. Absorção e redistribuição do nitrogênio aplicado via foliar em videiras jovens. Revista Brasileira de Fruticultura, Jaboticabal, v. 27, n. 1, p. 110-114, 2005.

CONRADIE, W. J. Distribution and translocation of nitrogen absorbed during early summer by two-year-old grapevines grown in sand culture. American Journal of Enology and Viticulture, Davis, v. 42, p. 180-190, 1991.

EMBRAPA - CNPS. Sistema brasileiro de classificação de solos. Rio de Janeiro: Embrapa Solos, 1999. 412 p.

FRACARO, A. A. Aplicação de ethephon em videira 'Niágara Rosada' (Vitis labrusca L.) visando a produção na entressafra do Estado de São Paulo. 2004. 79f. Tese 
(Doutorado em Produção Vegetal) - Faculdade de Ciência Agrárias e Veterinárias, Universidade Estadual Paulista "Júlio de Mesquita Filho", Jaboticabal, 2004.

FRACARO, A. A.; BOLIANI, A. C. Efeito do ethephon em videira 'Rubi' (Vitis vinifera L.), cultivada na região noroeste do Estado de São Paulo. Revista Brasileira de Fruticultura, Jaboticabal, v. 23, n.3, p. 510-512, 2001.

FRACARO, A. A.; PEREIRA, F. M. Efeito do ethephon sobre a brotação e vigor dos ramos da videira 'Niágara Rosada' (Vitis labrusca L.). Revista Brasileira de Fruticultura, Jaboticabal, v. 26, n. 3, p. 399-402, 2004.

FRACARO, A. A.; PEREIRA, F. M.; NACHTIGAL, J. C. Uso do ethephon antes da poda de produção em videira 'Niágara Rosada' (Vitis labrusca L.). Revista Brasileira de Fruticultura, Jaboticabal, v. 26, n. 1, p. 97-100, 2004a.

FRACARO, A. A.; PEREIRA, F. M.; NACHTIGAL, J. C.; BARBOSA, J. C. Efeito do ethephon sobre a produção da uva 'Niágara Rosada' (Vitis labrusca L.), produzida na entressafra na região de Jales-SP. Revista Brasileira de Fruticultura, Jaboticabal, v. 26, n. 1, p. 82-85, 2004 b.

MAJEROWICZ, N. Fotossíntes. In: KERBAUY, G.B. Fisiologia vegetal. Rio de Janeiro: Guanabara-Koogan, 2004. 114-178 p.

MARINHO, C. S.; TERRA, F. A.; MONNERAT, P. H.; CARVALHO, J. C. Desequilíbrio nutricional na limeira ácida tahiti induzido pela aplicação de ethephon. Revista Brasileira de Fruticultura, Jaboticabal, v.23, n.3, p.745-747, 2001.

MASHIMA, C.H. Uva sem semente. Recife: SEBRAE/PE, 2000. 51p. (Série Agricultura, 14)

PEREIRA, F. M.; FRACARO, A. A. Efeito do ethephon na qualidade da uva 'Niágara Rosada' (Vitis labrusca L.), produzida na entressafra, na região de Jales-SP. Revista Brasileira de Fruticultura, Jaboticabal, v. 26, n. 2, p. 254257, 2004.

POMMER, C. V. Uva tecnologia de produção, pós-colheita, mercado. Porto Alegre: Cinco Continentes, 2003. 778 p.

PSZCZOLKOWSKI, P. T. Problemas de mala brotación en condiciones tropicales. In: CURSO UVA DE MESA DE EXPORTACIÓN: PROBLEMAS DE PRODUCIÓN Y CALIDAD, 1984, Campos San Joaquim, Santiago, Chile. Resumenes... Santiago: Pontifícia Universidad Catolica de Chile, Faculdad de Agronomia, , 1984. p.14.1-14.11.

SANTOS, H. P.; PURGATTO, E.; MERCEIR, H.; BUCKERIDGE, M. S. The control of storage xyloglucan mobilization in cotyledons of Hymenaea courbaril. Plant Physiology, Rockville, v. 135, p. 287-299, 2004.

TAIZ, L.; ZEIGER, E. Fisiologia vegetal. Porto Alegre: Artmed, 2004. 719 p.

TEDESCO, M. J.; GIANELLO, C.; BISSANI, C. A.; BOHNEN, H.; VOLKWEISS, S. J. Análise de solo, plantas e outros materiais. Porto Alegre: UFRGS, 1995. $174 \mathrm{p}$.

ZAPATA, C.; DELÉENS, E.; CHAILlOU, S.; MAGNÉ, C. Partitioning and mobilization of starch and $\mathrm{N}$ reserves in grapevine (Vitis vinifera L.). Journal of Plant Physiology, Leipzig, v.161, p. 1.031-1.040, 2004. 